\title{
Simulation and sensitivities for a phased IceCube-Gen2 deployment
}

\section{The IceCube-Gen2 Collaboration}

(a complete list of authors can be found at the end of the proceedings)

E-mail: baclark@msu.edu, hallid15@msu.edu

The IceCube Neutrino Observatory opened the window on high-energy neutrino astronomy by confirming the existence of $\mathrm{PeV}$ astrophysical neutrinos and identifying the first compelling astrophysical neutrino source in the blazar TXS0506+056. Planning is underway to build an enlarged detector, IceCube-Gen2, which will extend measurements to higher energies, increase the rate of observed cosmic neutrinos and provide improved prospects for detecting fainter sources. IceCube-Gen2 is planned to have an extended in-ice optical array, a radio array at shallower depths for detecting ultra-high-energy ( $>100 \mathrm{PeV}$ ) neutrinos, and a surface component studying cosmic rays. In this contribution, we will discuss the simulation of the in-ice optical component of the baseline design of the IceCube-Gen 2 detector, which foresees the deployment of an additional $\sim 120$ new detection strings to the existing 86 in IceCube over $\sim 7$ Antarctic summer seasons. Motivated by the phased construction plan for IceCube-Gen2, we discuss how the reconstruction capabilities and sensitivities of the instrument are expected to progress throughout its deployment.

Corresponding authors: Brian A. Clark ${ }^{1 *}$, Robert Halliday ${ }^{1}$

${ }^{1}$ Michigan State University, East Lansing, MI, United States

* Presenter

$37^{\text {th }}$ International Cosmic Ray Conference (ICRC 2021)

July 12 th - 23rd, 2021

Online - Berlin, Germany 


\section{Introduction}

Neutrinos are unique messengers to the distant and high-energy reaches of the Universe. Unlike cosmic rays and gamma rays, neutrinos are chargeless and weakly interacting particles, capable of travelling in straight lines from their birthplace in astrophysical accelerators. The IceCube observatory opened the window on high-energy neutrino astronomy by detecting an astrophysical neutrino flux in 2013 [1]. IceCube has characterized the flux of astrophysical neutrinos, concluding it is consistent with an isotropic arrival direction distribution. A fit to the energy $(E)$ distribution of the form a single single power law $E^{-\gamma}$ yields a spectral index $2<\gamma<3$. It also appears the three neutrino flavors contribute roughly equally to the total flux [2-5]. In addition to measuring this diffuse flux of neutrinos, IceCube has also searched for the sources of neutrinos, identifying the first compelling evidence of a neutrino source in the blazar TXS0506+056 [6, 7]. The IceCube detector itself is an array of 5,160 photomultiplier tubes deployed instrumenting a cubic kilometer of clear glacial ice near the geographic South Pole, and is designed to detect the Cherenkov light emitted by charged particles produced in neutrino interactions in the ice.

To discover ultra-high-energy neutrinos above $10 \mathrm{PeV}$, better characterize the flux of astrophysical neutrinos with a larger sample size, and identify more neutrino sources, a new, larger detector is needed. To meet this need, the IceCube-Gen2 detector is under development [8]. IceCube-Gen2 will feature an extended in-ice optical array, which is the focus of this proceeding, as well as a new shallow-radio array for the detection of ultra-high energy neutrinos, and a surface array for studying cosmic rays and for providing a veto to downgoing atmospheric neutrinos. IceCube-Gen2 will play an important and complimentary role to other next-generation neutrinos telescopes-such as KM3NeT [9], Baikal-GVD [10], P-ONE [11], etc.-in characterizing the astrophysical neutrino flux and searching for sources of neutrinos.

The construction of the IceCube-Gen2 detector is expected to take approximately seven years. Similarly to IceCube, during the construction phase the Gen2 array can already deliver a compelling scientific program. We discuss how the science capabilities evolve with the partially constructed array. In Sec. 2 we describe in the enlarged optical component of the IceCube-Gen2 facility. In Sec. 3 we describe the simulation and performance of the detector, and in Sec. 4 we describe the resulting expected sensitivity. Finally, in Sec. 5 we review our results and discuss the outlook going forward.

\section{The Gen2-Optical Instrument}

In this section, we describe the enlarged optical array, which is shown in a "top-down" view in Fig. 1. The array consists of 120 new strings of optical modules (OMs) deployed in a "sunflower" pattern around the original 86 IceCube strings. The total volume of the Gen2-Optical detector is $\sim 8 \mathrm{~km}^{3}$, about an order of magnitude larger than the IceCube detector. As can be seen in Fig. 1, the lateral spacing between strings has been roughly doubled, from $125 \mathrm{~m}$ in IceCube to $240 \mathrm{~m}$. The optimization of the string spacing is the topic of another proceeding at this conference [12]. The vertical spacing between OMs is $17 \mathrm{~m}$, the same as in IceCube; however, the OMs are distributed across a slightly larger range of depths, from 1340-2700 $\mathrm{m}$ in depth, instead of 1446-2451 m. 


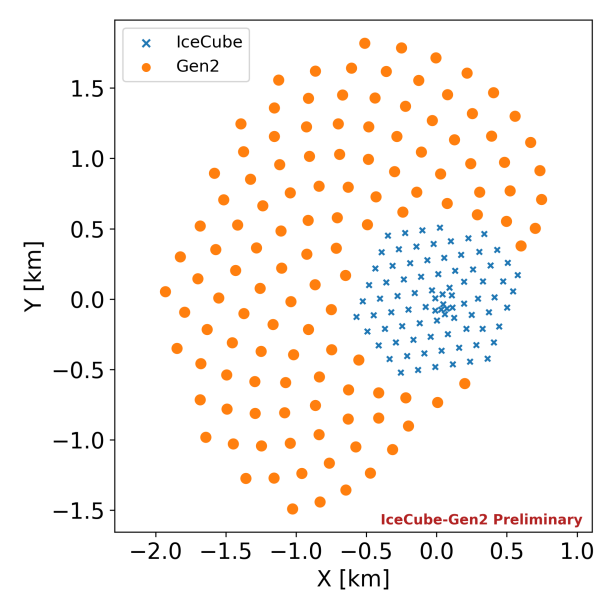

Figure 1: A top-down view of the IceCubeGen2 optical detector, with the original 86 IceCube strings marked with crosses, and the 120 new Gen 2 strings marked with circles.

Like in IceCube, neutrinos are expected in IceCube-Gen2 in two main light deposition patterns, or "morphologies;" both are visible in Fig. 2. The first, called "tracks," primarily arise from charged-current interactions of muon neutrinos, giving rise to long-lived daughter muons which cross the detector. The second, called "cascades," are roughly spherical depositions of light arising from neutral-current interactions of all flavors and charged-current interactions of electron and tau neutrinos. At sufficiently high energies $(\sim 1 \mathrm{PeV})$ tau neutrinos can also produce additional interesting morphologies; for example, the daughter tau can travel macroscopic distance from the primary vertex before decaying, resulting in a secondary deposition of light in a signature termed a "double bang". At EeV energies, the daughter taus become sufficiently long lived to appear as tracks.
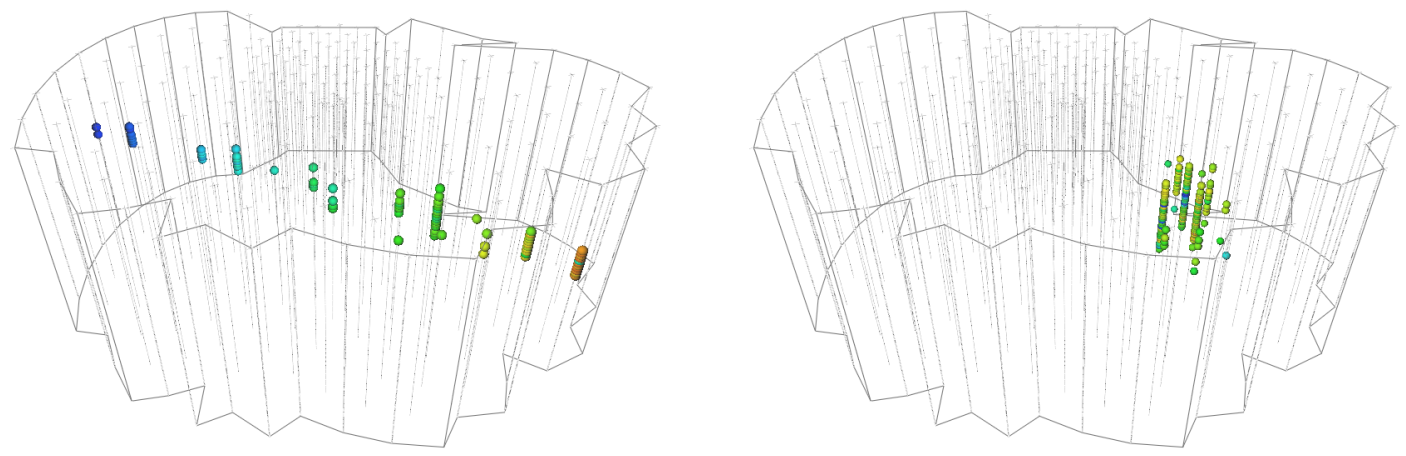

Figure 2: A view of the two main "morphologies" of neutrino interactions expected in the optical component of IceCube-Gen2: "tracks" (left) "cascades" (right). The size of a module is proportional to the quantity of the light recorded, while the color indicates the relative arrival time of the light (with red indicating earlier arrival times, and blue indicating later arrival times).

For these proceedings, we focus on the performance of the detector to a sub-class of events known as "throughgoing-tracks," as this class of events historically provides the best sensitivity in searches for neutrino sources [13]. Such events are largely produced by muons that cross the 


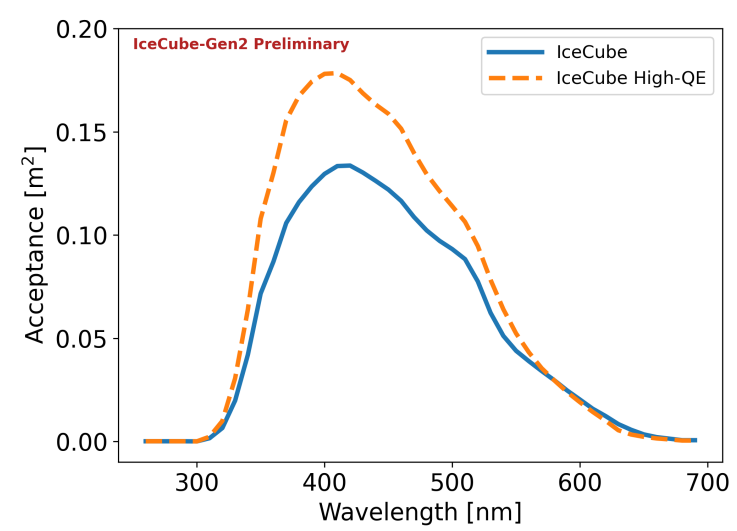

Figure 3: The acceptance to photons as a function of wavelength for two different types of optical modules used in IceCube: the standard IceCube OM, and a high quantum-efficiency variant used in DeepCore.

detector. The muons can either arise from astrophysical and atmospheric neutrinos interacting outside of the detector volume in both hemispheres, or from atmospheric muons in the southern hemisphere.

\section{Simulation and Performance}

In this section, we describe the process of simulating the optical component of IceCubeGen2 from particle generation, through photon propogation, detector simulation, processing and reconstruction. From this simulation we extract relevant performance metrics, specifically the effective area and pointing resolution.

\subsection{Simulation and Reconstruction}

The first step of our simulation is to specify the primary particle that will be generated. Since we focus on throughgoing tracks, we simulate primaries as single high-energy muons. These muons are injected with the MUONGUN [14] simulation package, and then further propagated with PROPOSAL [15], which estimates the energy depositions of the muons from continuous and stochastic processes such as ionization and bremsstrahlung. Muons are simulated as arriving isotropically from all directions on the sky and are distributed in energy between $3 \mathrm{TeV}$ and $100 \mathrm{PeV}$ according to a power law $E^{-1.4}$ spectrum; the hard spectral index ensures sufficient statistics to characterize the detector performance at high energies.

After energy depositions are specified, photons resulting from the interactions are propagated with either the CLSim or PPC package [16], taking into account scattering and attenuation from the ice. The intensity of Cherenkov photons goes as $1 / \lambda^{2}$ (where $\lambda$ is the wavelength of light), and so the spectrum is peaked in the ultra-violet; however, the response of the OMs is not, as can be seen in Fig. 3. As such, to be computational efficient, photons are drawn from the Cherenkov spectrum weighted by the wavelength acceptance of the OMs. Because the ray tracing does not know ahead of time which type of module a photon will encounter, photons are generated according to an envelope which encompasses the acceptance of all modules in the simulation. After arriving at a specific module, the photons are downsampled according to the acceptance and geometry of that specific module. 
After the photons arrive at the module, they must be converted into measured photoelectrons. For OMs in the original 86 IceCube strings, we apply the full IceCube simulation and pulse unfolding process: the photons are convolved with the response of the PMT and electronics, resulting in waveforms; those waveforms are then deconvolved back into a reconstructed pulse series. OMs for IceCube-Gen2 are in the conceptual design phase, hence no full PMT or electronics simulation is yet available. Instead, the photoelectrons are binned in time (mimicking the effects of a digitizer) and converted directly into reconstructed pulses.

Finally, a likelihood based reconstruction is applied to estimate the direction and energy of the muon. The method compares the observed number of photons to that expected for a hypothetical incident muon with incoming direction $\theta, \phi$ and energy $E$, and minimizes the difference in a likelihood to identify a best-fit hypothesis [17].

\subsection{Performance}

For evaluating detector performance, we focus on two metrics that drive the sensitivity of analyses utilizing throughgoing tracks, e.g. astrophysical neutrino source searches. These are the effective area and the angular resolution. We begin by applying cuts to identify well-reconstructed tracks. This is done by imposing requirements on the reconstructions, such as requiring a minimum number of hit OMs, a minimum reconstructed track length, etc. The energy $(E)$ and zenith $(\theta)$ dependent efficiency for passing these cuts defines the selection efficiency: $\eta(E, \theta)$.

To calculate the muon effective area in a given energy and zenith bin, we take the product of the projected geometric area of the detector $A_{\text {geo }}(\theta)$ and the selection efficiency $\eta(E, \theta) . A_{\text {geo }}(\theta)$ is found by placing a convex hull around the strings, defining a solid. To calculate the angular resolution, we apply the event selection, and then calculate the opening angle, or the difference between the reconstructed and true direction $\Delta \Psi$. The distribution of $\Delta \Psi$ defines the point spread function of the detector, which is parameterized as a function of energy and zenith using the "Moffat/King" function, as is done in e.g. the Fermi gamma ray telescope [18-20]. The full parameterization $\Delta \Psi(E, \theta)$ is needed for sensitivity studies in Sec. 4 . We take the median opening angle, $\Delta \Psi_{\text {med }}$, as the angular resolution.

The two metrics, muon effective area and angular resolution, are plotted in Fig. 4. They are plotted as a function of deployment season, and both are shown for two different energies and two different declinations; for context, we also show the performance of the IceCube detector at one representative choice of declination and energy. We assume a schedule for deployment where an equal number of strings are deployed each year. As can be seen, even by the middle of the scheduled deployment process, the total aperture for horizontal events, which provide the best reconstruction performance, will have increased by more than a factor of two, and the angular resolution will have improved by $\sim 50 \%$. To be conservative, these metrics do not factor in the optical modules enhanced directional reconstruction capabilities from having directional light sensing capabilities through multiple PMTs, a major improvement over IceCube DOMs.

\section{Sensitivity}

In assessing the sensitivity of the detector, we continue to focus our science goals on the search for sources of astrophysical neutrinos. We quantify this in two different ways: a) the time-integrated 

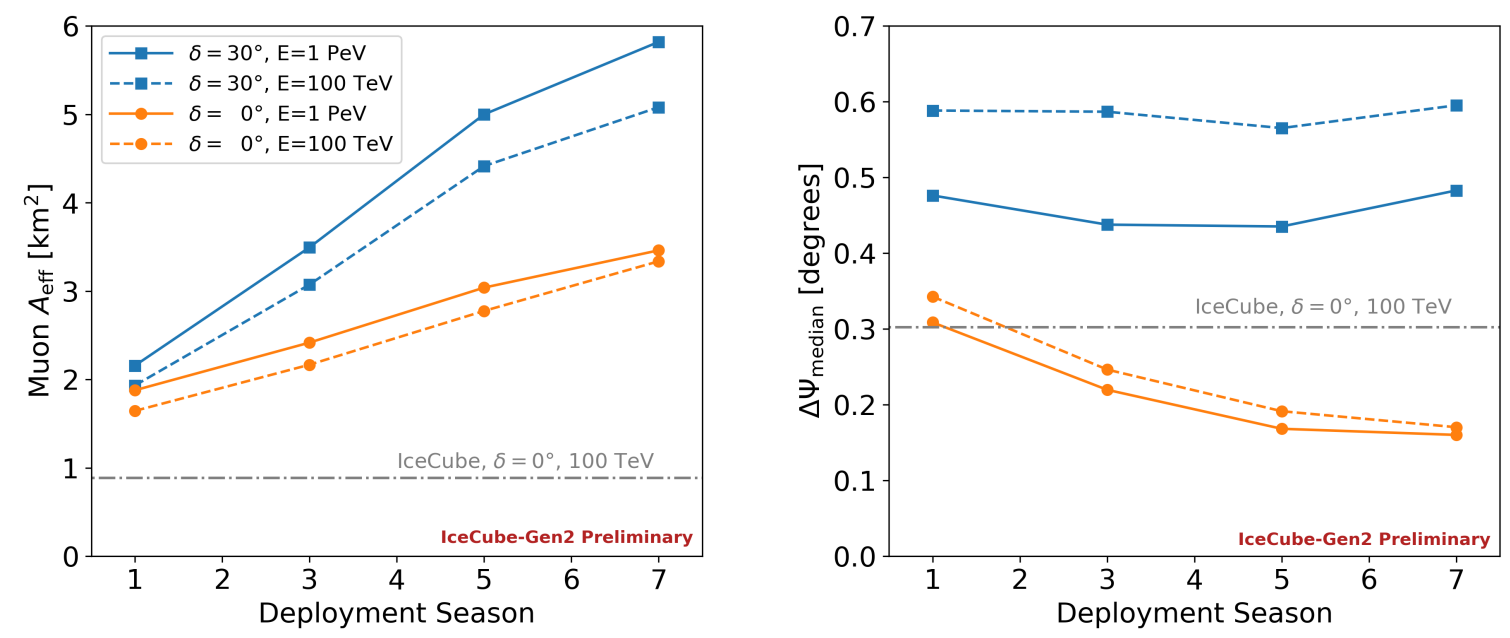

Figure 4: Plots of two metrics of the performance of the detector performance: the muon effective area (left) and the angular resolution (right) as a function of time for several choices of muon energy and declination.

sensitivity of the detector to a steady point-source of neutrinos (subsection 4.1) and b) the sensitivity of the detector to a flaring source of neutrinos (subsection 4.2). For both, we take the background to the neutrino search as the sum of the conventional (Honda 2006 [21]) and prompt (Enberg [22]) atmospheric neutrino fluxes with knee reweighting according to Gaisser H3a [23].

\subsection{Steady Sources}

In modeling the sensitivity of the detector to steady point sources, we calculate the discovery potential of a mock time-integrated search. We define the discovery potential as the minimum value required of a parameter of the astrophysical neutrino flux (typically the normalization), such that an experimental search would exclude the parameter as being zero (the null hypothesis) at the $5 \sigma$ level. For purposes of comparing the capabilities as a function of time, we calculate the discovery potential assuming a time integration of one year. The resulting discovery potential for IceCube-Gen2 is shown in Fig. 5; we also show the discovery potential of the existing IceCube detector for reference. We emphasize that one year time-integrated discovery potentials are only shown for purpose of comparison, as the achieved discovery potential in each season will be significantly better if it is combined with the previous years data. The discovery potential is shown for the normalization on the flux of neutrinos, assuming an unbroken power law with a spectral index of -2 .

As in IceCube, the sensitivity of the detector is better for sources located in the Northern Sky $(\sin \delta>0)$, where the Earth serves as a shield to the flux of atmospheric muons. IceCube-Gen2 also improves on the performance of IceCube near the Celestial South Pole, where an enlarged surface veto enables rejection of vertically downgoing atmospheric air showers over a broader range in zeniths. This can be seen as a flattening of the discovery potential (relative to IceCube) at $\sin \delta<-0.5$ in Fig. 5, and is discussed further elsewhere [8]. As the deployment seasons progress, the discovery potential lowers to see approximately $\sim 4$ times fainter sources. The sensitivity will be further improved by the multi-PMT IceCube-Gen2 optical modules, as previously mentioned in 3.2 , which we have not included in these estimates. 

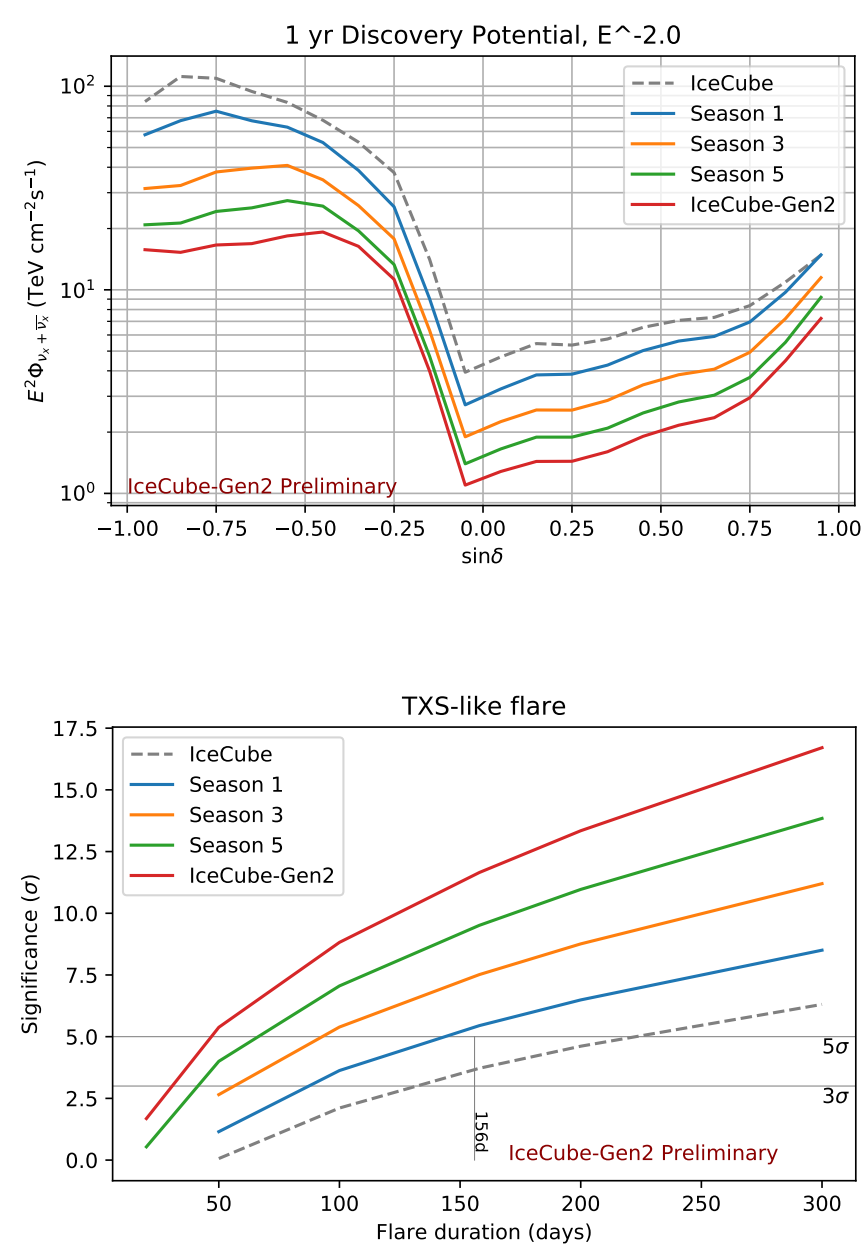

Figure 5: One-year time-integrated discovery potentials for a steady point source of neutrinos with a unbroken power law spectrum $E^{-2}$. Each line indicates a different year of IceCube-Gen2 construction. Also shown for reference is the 1year discovery potential of IceCube. We emphasize that one year time-integrated discovery potentials are only shown for purpose of comparison, as the achieved discovery potential in each season will be significantly better if it is combined with the previous years data.

Figure 6: Significance as a function of flare duration for various stages of IceCube-Gen 2 construction including trials correction. Backgrounds here are calculated from the previously mentioned atmospheric and prompt fluxes, summed with an additional diffuse $E^{-2}$ astrophysical background component. Vertical bar in the center marks the measured flare duration in IceCube. IceCube significance included for reference.

\subsection{Flaring Sources}

To calculate the sensitivity of the detector to a flaring source of neutrinos, we consider the detection significance of a flare in IceCube-Gen2 as a function of flare duration. We take as a template the fluence of the 2014-15 flare of neutrinos from TXS0506+056, which had a measured spectral index of $\gamma=2.2 \pm 0.2$. We vary the flare duration, and calculate the significance with which it would be recorded in the various seasons of IceCube-Gen2 deployment. This "time-tosignificance" can be seen in Fig. 6, where each line indicates a different year of the IceCube-Gen2 construction process; for reference, the bottom line shows IceCube. For a TXS-like flare, which lasted 156 days in the assumption of a box-shaped time window for the emissions, the flare could have been detected in IceCube-Gen2 at greater than $5 \sigma$ significance after just the first or second season of construction (given the deployment scheduled assumed in this proceedings, where an equal number of strings is deployed each season). Importantly, this detection could be made using the neutrino search only, without any multimessenger gamma-ray counterparts, suggesting that IceCube-Gen2 will be sensitive to so called "hidden sources" which might live in gamma-ray opaque environments. 


\section{Conclusion and Outlook}

In this proceeding, we have described the IceCube-Gen2 optical instrument, and the simulation process to determine sensitivities. Motivated by the $\sim 7$ year deployment schedule, we determined the performance and sensitivity of the detector over time. For the performance, we focused on throughgoing tracks, which provide the best discovery potential for astrophysical sources. We have evaluated the effective area and angular resolution of such events, finding an approximately four times greater effective area and an approximately two times improved angular resolution. Finally, we show the projected sensitivity of the detector to both steady and flaring sources of neutrinos, showing that in the time that IceCube observed the TXS flare at $3.5 \sigma$, the full IceCube-Gen2 detector would have seen it at more than $10 \sigma$. These performance characteristics show that even during construction, IceCube-Gen2 will have a rapidly increasing discovery potential for astrophysical neutrino sources and will be essential for multi-messenger science.

\section{References}

[1] IceCube Collaboration, M. G. Aartsen et al. Science 342 (2013) 1242856.

[2] IceCube Collaboration, R. Abbasi et al. Phys. Rev. D 104 (Jul, 2021) 022002.

[3] IceCube Collaboration, M. G. Aartsen et al. Phys. Rev. Lett. 125 no. 12, (2020) 121104.

[4] IceCube Collaboration, M. G. Aartsen et al. Phys. Rev. D 99 no. 3, (2019) 032004.

[5] IceCube Collaboration, J. Stettner PoS ICRC2019 (2020) 1017.

[6] IceCube, Fermi-LAT, MAGIC, AGILE, ASAS-SN, HAWC, H.E.S.S., INTEGRAL, Kanata, Kiso, Kapteyn, Liverpool Telescope, Subaru, Swift NuSTAR, VERITAS, VLA/17B-403 Collaboration, M. G. Aartsen et al. Science 361 no. 6398, (2018) eaat1378.

[7] IceCube Collaboration, M. G. Aartsen et al. Science 361 no. 6398, (2018) 147-151.

[8] IceCube-Gen2 Collaboration, M. G. Aartsen et al. J. Phys. G 48 no. 6, (2021) 060501.

[9] KM3Net Collaboration, S. Adrian-Martinez et al. J. Phys. G 43 no. 8, (2016) 084001.

[10] I. A. Belolaptikov et al. Astroparticle Physics 7 no. 3, (Aug., 1997) 263-282.

[11] P-ONE Collaboration, M. Agostini et al. Nature Astron. 4 no. 10, (2020) 913-915.

[12] IceCube Collaboration, A. Omeliukh PoS ICRC2021 (these proceedings) 1184.

[13] IceCube Collaboration, M. G. Aartsen et al. Phys. Rev. Lett. 124 no. 5, (2020) 051103.

[14] J. van Santen, Neutrino Interactions in IceCube above $1 \mathrm{TeV}$. PhD thesis, University of Wisconsin-Madison, 2014.

[15] J. H. Koehne et al. Comput. Phys. Commun. 184 (2013) 2070-2090.

[16] D. Chirkin et al., "Photon propagation using gpus by the icecube neutrino observatory," in 2019 15th International Conference on eScience (eScience), pp. 388-393. 2019.

[17] IceCube Collaboration, M. G. Aartsen et al. JINST 9 (2014) P03009.

[18] A. F. J. Moffat Astronomy and Astrophysics 3 (Dec., 1969) 455.

[19] I. King Astronomical Journal 67 (Oct., 1962) 471.

[20] "The point spread function." https://fermi.gsfc.nasa.gov/ssc/data/analysis/ documentation/Cicerone/Cicerone_LAT_IRFs/IRF_PSF.html, 2018. Accessed: 2021-05-28.

[21] M. Honda et al. Phys. Rev. D 75 (2007) 043006.

[22] R. Enberg, M. H. Reno, and I. Sarcevic Phys. Rev. D 78 (2008) 043005.

[23] T. K. Gaisser Astropart. Phys. 35 (2012) 801-806. 


\section{Full Author List: IceCube-Gen2 Collaboration}

R. Abbasi ${ }^{17}$, M. Ackermann ${ }^{71}$, J. Adams ${ }^{22}$, J. A. Aguilar ${ }^{12}$, M. Ahlers ${ }^{26}$, M. Ahrens ${ }^{60}$, C. Alispach ${ }^{32}$, P. Allison ${ }^{24,25}$, A. A. Alves $\mathrm{Jr}^{35}$, N. M. Amin ${ }^{50}$, R. An ${ }^{14}$, K. Andeen ${ }^{48}$, T. Anderson ${ }^{67}$, G. Anton ${ }^{30}$, C. Argüelles ${ }^{14}$, T. C. Arlen ${ }^{67}$, Y. Ashida ${ }^{45}$, S. Axani ${ }^{15}, \mathrm{X}$. Bai $^{56}$, A. Balagopal V. ${ }^{45}$, A. Barbano ${ }^{32}$, I. Bartos ${ }^{52}$, S. W. Barwick ${ }^{34}$, B. Bastian ${ }^{71}$, V. Basu ${ }^{45}$, S. Baur ${ }^{12}$, R. Bay ${ }^{8}$, J. J. Beatty ${ }^{24,} 25$, K.-H. Becker ${ }^{70}$, J. Becker Tjus ${ }^{11}$, C. Bellenghi ${ }^{31}$, S. BenZvi ${ }^{58}$, D. Berley ${ }^{23}$, E. Bernardini ${ }^{71,72}$, D. Z. Besson ${ }^{38,73}$, G. Binder ${ }^{8,9}$, D. Bindig $^{70}$, A. Bishop ${ }^{45}$, E. Blaufuss ${ }^{23}$, S. Blot ${ }^{71}$, M. Boddenberg ${ }^{1}$, M. Bohmer ${ }^{31}$, F. Bontempo ${ }^{35}$, J. Borowka ${ }^{1}$, S. Böser ${ }^{46}$, O. Botner ${ }^{69}$, J. Böttcher ${ }^{1}$, E. Bourbeau ${ }^{26}$, F. Bradascio ${ }^{71}$, J. Braun ${ }^{45}$, S. Bron ${ }^{32}$, J. Brostean-Kaiser ${ }^{71}$, S. Browne ${ }^{36}$, A. Burgman ${ }^{69}$, R. T. Burley ${ }^{2}$, R. S. Busse ${ }^{49}$, M. A. Campana ${ }^{55}$, E. G. Carnie-Bronca ${ }^{2}$, M. Cataldo ${ }^{30}$, C. Chen ${ }^{6}$, D. Chirkin ${ }^{45}$, K. Choi ${ }^{62}$, B. A. Clark ${ }^{28}$, K. Clark ${ }^{37}$, R. Clark $^{40}$, L. Classen ${ }^{49}$, A. Coleman ${ }^{50}$, G. H. Collin ${ }^{15}$, A. Connolly ${ }^{24}$, 25 , J. M. Conrad ${ }^{15}$, P. Coppin ${ }^{13}$, P. Correa ${ }^{13}$, D. F. Cowen ${ }^{66,67}$, R. $\mathrm{Cross}^{58}$, C. Dappen ${ }^{1}$, P. Dave ${ }^{6}$, C. Deaconu ${ }^{20,21}$, C. De Clercq ${ }^{13}$, S. De Kockere ${ }^{13}$, J. J. DeLaunay ${ }^{67}$, H. Dembinski ${ }^{50}$, K. Deoskar ${ }^{60}$, S. De Ridder ${ }^{33}$, A. Desai ${ }^{45}$, P. Desiati ${ }^{45}$, K. D. de Vries ${ }^{13}$, G. de Wasseige ${ }^{13}$, M. de With ${ }^{10}$, T. DeYoung ${ }^{28}$, S. Dharani ${ }^{1}$, A. Diaz $^{15}$, J. C. Díaz-Vélez ${ }^{45}$, M. Dittmer ${ }^{49}$, H. Dujmovic ${ }^{35}$, M. Dunkman ${ }^{67}$, M. A. DuVernois ${ }^{45}$, E. Dvorak ${ }^{56}$, T. Ehrhardt ${ }^{46}$, P. Eller ${ }^{31}$, R. Engel $^{35,36}$, H. Erpenbeck ${ }^{1}$, J. Evans ${ }^{23}$, J. J. Evans ${ }^{47}$, P. A. Evenson ${ }^{50}$, K. L. Fan ${ }^{23}$, K. Farrag ${ }^{41}$, A. R. Fazely ${ }^{7}$, S. Fiedlschuster ${ }^{30}$, A. T. Fienberg ${ }^{67}$, K. Filimonov ${ }^{8}$, C. Finley ${ }^{60}$, L. Fischer ${ }^{71}$, D. Fox ${ }^{66}$, A. Franckowiak ${ }^{11,71}$, E. Friedman ${ }^{23}$, A. Fritz ${ }^{46}$, P. Fürst ${ }^{1}$, T. K. Gaisser ${ }^{50}$, J. Gallagher ${ }^{44}$, E. Ganster ${ }^{1}$, A. Garcia ${ }^{14}$, S. Garrappa ${ }^{71}$, A. Gartner ${ }^{31}$, L. Gerhardt ${ }^{9}$, R. Gernhaeuser ${ }^{31}$, A. Ghadimi ${ }^{65}$, P. Giri ${ }^{39}$, C. Glaser ${ }^{69}$, T. Glauch ${ }^{31}$, T. Glüsenkamp ${ }^{30}$, A. Goldschmidt ${ }^{9}$, J. G. Gonzalez ${ }^{50}$, S. Goswami ${ }^{65}$, D. Grant ${ }^{28}$, T. Grégoire ${ }^{67}$, S. Griswold ${ }^{58}$, M. Gündüz ${ }^{11}$, C. Günther ${ }^{1}$, C. Haack ${ }^{31}$, A. Hallgren ${ }^{69}$, R. Halliday ${ }^{28}$, S. Hallmann ${ }^{71}$, L. Halve ${ }^{1}$, F. Halzen ${ }^{45}$, M. $\mathrm{Ha} \mathrm{Minh}^{31}$, K. Hanson ${ }^{45}$, J. Hardin ${ }^{45}$, A. A. Harnisch ${ }^{28}$, J. Haugen ${ }^{45}$, A. Haungs ${ }^{35}$, S. Hauser ${ }^{1}$, D. Hebecker ${ }^{10}$, D. Heinen ${ }^{1}$, K.

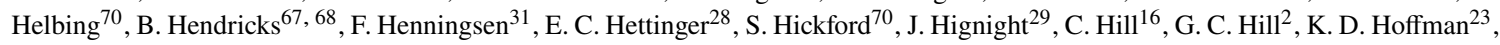
B. Hoffmann ${ }^{35}$, R. Hoffmann ${ }^{70}$, T. Hoinka ${ }^{27}$, B. Hokanson-Fasig ${ }^{45}$, K. Holzapfel ${ }^{31}$, K. Hoshina ${ }^{45,64}$, F. Huang ${ }^{67}$, M. Huber ${ }^{31}$, T.

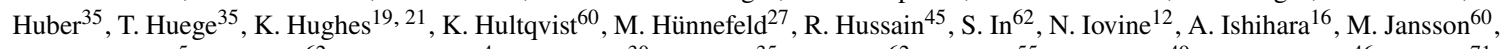
G. S. Japaridze ${ }^{5}$, M. Jeong ${ }^{62}$, B. J. P. Jones ${ }^{4}$, O. Kalekin ${ }^{30}$, D. Kang ${ }^{35}$, W. Kang ${ }^{62}$, X. Kang ${ }^{55}$, A. Kappes ${ }^{49}$, D. Kappesser ${ }^{46}$, T. Karg ${ }^{71}$, M. Karl ${ }^{31}$, A. Karle ${ }^{45}$, T. Katori ${ }^{40}$, U. Katz ${ }^{30}$, M. Kauer ${ }^{45}$, A. Keivani ${ }^{52}$, M. Kellermann ${ }^{1}$, J. L. Kelley ${ }^{45}$, A. Kheirandish ${ }^{67}$, K. Kin ${ }^{16}$, T. Kintscher ${ }^{71}$, J. Kiryluk ${ }^{61}$, S. R. Klein ${ }^{8,9}$, R. Koirala ${ }^{50}$, H. Kolanoski ${ }^{10}$, T. Kontrimas ${ }^{31}$, L. Köpke ${ }^{46}$, C. Kopper ${ }^{28}$, S. Kopper ${ }^{65}$, D. J. Koskinen ${ }^{26}$, P. Koundal ${ }^{35}$, M. Kovacevich ${ }^{55}$, M. Kowalski ${ }^{10,71}$, T. Kozynets ${ }^{26}$, C. B. Krauss ${ }^{29}$, I. Kravchenko ${ }^{39}$, R. Krebs ${ }^{67}{ }^{68}$, E.

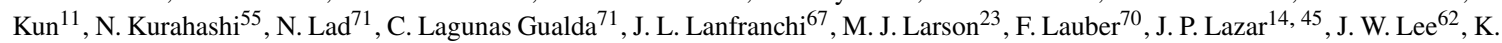

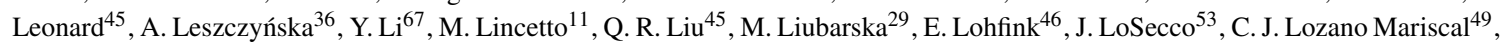
L. $\mathrm{Lu}^{45}$, F. Lucarelli ${ }^{32}$, A. Ludwig ${ }^{28,42}$, W. Luszczak ${ }^{45}$, Y. Lyu ${ }^{8,9}$, W. Y. Ma ${ }^{71}$, J. Madsen ${ }^{45}$, K. B. M. Mahn ${ }^{28}$, Y. Makino ${ }^{45}$, S. Mancina $^{45}$, S. Mandalia ${ }^{41}$, I. C. Mariş ${ }^{12}$, S. Marka ${ }^{52}$, Z. Marka ${ }^{52}$, R. Maruyama ${ }^{51}$, K. Mase ${ }^{16}$, T. McElroy ${ }^{29}$, F. McNally ${ }^{43}$, J. V. Mead $^{26}$, K. Meagher ${ }^{45}$, A. Medina ${ }^{25}$, M. Meier ${ }^{16}$, S. Meighen-Berger ${ }^{31}$, Z. Meyers ${ }^{71}$, J. Micallef ${ }^{28}$, D. Mocklerr ${ }^{12}$, T. Montaruli ${ }^{32}$, R. W. Moore ${ }^{29}$, R. Morse ${ }^{45}$, M. Moulai ${ }^{15}$, R. Naab ${ }^{71}$, R. Nagai ${ }^{16}$, U. Naumann ${ }^{70}$, J. Necker ${ }^{71}$, A. Nelles ${ }^{30,71}$, L. V. Nguyê̂n ${ }^{28}, H$. Niederhausen $^{31}$, M. U. Nisa ${ }^{28}$, S. C. Nowicki ${ }^{28}$, D. R. Nygren ${ }^{9}$, E. Oberla ${ }^{20,21}$, A. Obertacke Pollmann ${ }^{70}$, M. Oehler ${ }^{35}$, A. Olivas ${ }^{23}$, A. Omeliukh ${ }^{71}$, E. O'Sullivan ${ }^{69}$, H. Pandya ${ }^{50}$, D. V. Pankova ${ }^{67}$, L. Papp ${ }^{31}$, N. Park ${ }^{37}$, G. K. Parker ${ }^{4}$, E. N. Paudel ${ }^{50}$, L. Paul ${ }^{48}$, C. Pérez de $\operatorname{los} \operatorname{Heros}^{69}$, L. Peters ${ }^{1}$, T. C. Petersen ${ }^{26}$, J. Peterson ${ }^{45}$, S. Philippen ${ }^{1}$, D. Pieloth ${ }^{27}$, S. Pieper ${ }^{70}$, J. L. Pinfold ${ }^{29}$, M. Pittermann ${ }^{36}$, A. Pizzuto $^{45}$, I. Plaisier ${ }^{71}$, M. Plum ${ }^{48}$, Y. Popovych ${ }^{46}$, A. Porcelli ${ }^{33}$, M. Prado Rodriguez ${ }^{45}$, P. B. Price ${ }^{8}$, B. Pries ${ }^{28}$, G. T. Przybylski ${ }^{9}$, L. Pyras $^{71}$, C. Raab ${ }^{12}$, A. Raissi ${ }^{22}$, M. Rameez ${ }^{26}$, K. Rawlins ${ }^{3}$, I. C. Rea ${ }^{31}$, A. Rehman ${ }^{50}$, P. Reichherzer ${ }^{11}$, R. Reimann ${ }^{1}$, G. Renzi ${ }^{12}$, E. Resconi ${ }^{31}$, S. Reusch ${ }^{71}$, W. Rhode 27 , M. Richman ${ }^{55}$, B. Riedel ${ }^{45}$, M. Riegel ${ }^{35}$, E. J. Roberts ${ }^{2}$, S. Robertson ${ }^{8,9}$, G. Roellinghoff ${ }^{62}$, M. Rongen ${ }^{46}$, C. Rott ${ }^{59}$ 62, T. Ruhe ${ }^{27}$, D. Ryckbosch ${ }^{33}$, D. Rysewyk Cantu ${ }^{28}$, I. Safa ${ }^{14,45}$, J. Saffer ${ }^{36}$, S. E. Sanchez Herrera ${ }^{28}$, A. Sandrock $^{27}$, J. Sandroos ${ }^{46}$, P. Sandstrom ${ }^{45}$, M. Santander ${ }^{65}$, S. Sarkar ${ }^{54}$, S. Sarkar ${ }^{29}$, K. Satalecka ${ }^{71}$, M. Scharf ${ }^{1}$, M. Schaufel ${ }^{1}$, H. Schieler $^{35}$, S. Schindler ${ }^{30}$, P. Schlunder ${ }^{27}$, T. Schmidt ${ }^{23}$, A. Schneider ${ }^{45}$, J. Schneider ${ }^{30}$, F. G. Schröder ${ }^{35}$, 50, L. Schumacher ${ }^{31}$, G. Schwefer $^{1}$, S. Sclafani ${ }^{55}$, D. Seckel ${ }^{50}$, S. Seunarine ${ }^{57}$, M. H. Shaevitz ${ }^{52}$, A. Sharma ${ }^{69}$, S. Shefali ${ }^{36}$, M. Silva ${ }^{45}$, B. Skrzypek ${ }^{14}$, D. Smith $^{19,21}$, B. Smithers ${ }^{4}$, R. Snihur ${ }^{45}$, J. Soedingrekso ${ }^{27}$, D. Soldin ${ }^{50}$, S. Söldner-Rembold ${ }^{47}$, D. Southall ${ }^{19,21}$, C. Spannfellner ${ }^{31}$, G. M. Spiczak ${ }^{57}$, C. Spiering ${ }^{71}$, 73, J. Stachurska ${ }^{71}$, M. Stamatikos ${ }^{25}$, T. Stanev ${ }^{50}$, R. Stein ${ }^{71}$, J. Stettner ${ }^{1}$, A. Steuer ${ }^{46}$, T. Stezelberger ${ }^{9}$, T.

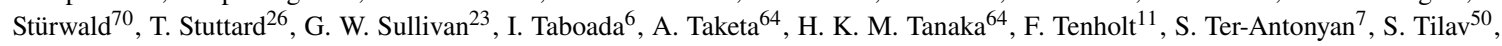
F. Tischbein ${ }^{1}$, K. Tollefson ${ }^{28}$, L. Tomankova ${ }^{11}$, C. Tönnis ${ }^{63}$, J. Torres ${ }^{24,25}$, S. Toscano ${ }^{12}$, D. Tosi ${ }^{45}$, A. Trettin ${ }^{71}$, M. Tselengidou ${ }^{30}$, C. F. Tung ${ }^{6}$, A. Turcati ${ }^{31}$, R. Turcotte ${ }^{35}$, C. F. Turley ${ }^{67}$, J. P. Twagirayezu ${ }^{28}$, B. Ty $^{45}$, M. A. Unland Elorrieta ${ }^{49}$, N. Valtonen-Mattila ${ }^{69}$, J. Vandenbroucke ${ }^{45}$, N. van Eijndhoven ${ }^{13}$, D. Vannerom ${ }^{15}$, J. van Santen $^{71}$, D. Veberic ${ }^{35}$, S. Verpoest ${ }^{33}$, A. Vieregg ${ }^{18,}$ 19, 20, 21, M. Vraeghe $^{33}$, C. Walck ${ }^{60}$, T. B. Watson ${ }^{4}$, C. Weaver ${ }^{28}$, P. Weigel ${ }^{15}$, A. Weindl ${ }^{35}$, L. Weinstock ${ }^{1}$, M. J. Weiss ${ }^{67}$, J. Weldert ${ }^{46}$, C. Welling ${ }^{71}$, C. Wendt ${ }^{45}$, J. Werthebach ${ }^{27}$, M. Weyrauch ${ }^{36}$, N. Whitehorn ${ }^{28,}{ }^{42}$, C. H. Wiebusch ${ }^{1}$, D. R. Williams ${ }^{65}$, S. Wissel ${ }^{66,67,68}$, M. Wolf $^{31}$, K. Woschnagg ${ }^{8}$, G. Wrede ${ }^{30}$, S. Wren ${ }^{47}$, J. Wulff ${ }^{11}$, X. W. Xu ${ }^{7}$, Y. Xu ${ }^{61}$, J. P. Yanez ${ }^{29}$, S. Yoshida ${ }^{16}$, S. Yu ${ }^{28}$, T. Yuan ${ }^{45}$, Z. Zhang ${ }^{61}$, S. Zierke ${ }^{1}$

\footnotetext{
${ }^{1}$ III. Physikalisches Institut, RWTH Aachen University, D-52056 Aachen, Germany

${ }^{2}$ Department of Physics, University of Adelaide, Adelaide, 5005, Australia

${ }^{3}$ Dept. of Physics and Astronomy, University of Alaska Anchorage, 3211 Providence Dr., Anchorage, AK 99508, USA

${ }^{4}$ Dept. of Physics, University of Texas at Arlington, 502 Yates St., Science Hall Rm 108, Box 19059, Arlington, TX 76019, USA

${ }^{5}$ CTSPS, Clark-Atlanta University, Atlanta, GA 30314, USA

${ }^{6}$ School of Physics and Center for Relativistic Astrophysics, Georgia Institute of Technology, Atlanta, GA 30332, USA

${ }^{7}$ Dept. of Physics, Southern University, Baton Rouge, LA 70813, USA

${ }^{8}$ Dept. of Physics, University of California, Berkeley, CA 94720, USA
} 
${ }^{9}$ Lawrence Berkeley National Laboratory, Berkeley, CA 94720, USA

${ }^{10}$ Institut für Physik, Humboldt-Universität zu Berlin, D-12489 Berlin, Germany

${ }^{11}$ Fakultät für Physik \& Astronomie, Ruhr-Universität Bochum, D-44780 Bochum, Germany

${ }^{12}$ Université Libre de Bruxelles, Science Faculty CP230, B-1050 Brussels, Belgium

${ }^{13}$ Vrije Universiteit Brussel (VUB), Dienst ELEM, B-1050 Brussels, Belgium

${ }^{14}$ Department of Physics and Laboratory for Particle Physics and Cosmology, Harvard University, Cambridge, MA 02138, USA

${ }^{15}$ Dept. of Physics, Massachusetts Institute of Technology, Cambridge, MA 02139, USA

${ }^{16}$ Dept. of Physics and Institute for Global Prominent Research, Chiba University, Chiba 263-8522, Japan

${ }^{17}$ Department of Physics, Loyola University Chicago, Chicago, IL 60660, USA

${ }^{18}$ Dept. of Astronomy and Astrophysics, University of Chicago, Chicago, IL 60637, USA

${ }^{19}$ Dept. of Physics, University of Chicago, Chicago, IL 60637, USA

${ }^{20}$ Enrico Fermi Institute, University of Chicago, Chicago, IL 60637, USA

${ }^{21}$ Kavli Institute for Cosmological Physics, University of Chicago, Chicago, IL 60637, USA

22 Dept. of Physics and Astronomy, University of Canterbury, Private Bag 4800, Christchurch, New Zealand

${ }^{23}$ Dept. of Physics, University of Maryland, College Park, MD 20742, USA

${ }^{24}$ Dept. of Astronomy, Ohio State University, Columbus, OH 43210, USA

${ }^{25}$ Dept. of Physics and Center for Cosmology and Astro-Particle Physics, Ohio State University, Columbus, OH 43210, USA

${ }^{26}$ Niels Bohr Institute, University of Copenhagen, DK-2100 Copenhagen, Denmark

${ }^{27}$ Dept. of Physics, TU Dortmund University, D-44221 Dortmund, Germany

${ }^{28}$ Dept. of Physics and Astronomy, Michigan State University, East Lansing, MI 48824, USA

${ }^{29}$ Dept. of Physics, University of Alberta, Edmonton, Alberta, Canada T6G 2E1

${ }^{30}$ Erlangen Centre for Astroparticle Physics, Friedrich-Alexander-Universität Erlangen-Nürnberg, D-91058 Erlangen, Germany

${ }^{31}$ Physik-department, Technische Universität München, D-85748 Garching, Germany

${ }^{32}$ Département de physique nucléaire et corpusculaire, Université de Genève, CH-1211 Genève, Switzerland

${ }^{33}$ Dept. of Physics and Astronomy, University of Gent, B-9000 Gent, Belgium

${ }^{34}$ Dept. of Physics and Astronomy, University of California, Irvine, CA 92697, USA

${ }^{35}$ Karlsruhe Institute of Technology, Institute for Astroparticle Physics, D-76021 Karlsruhe, Germany

${ }^{36}$ Karlsruhe Institute of Technology, Institute of Experimental Particle Physics, D-76021 Karlsruhe, Germany

${ }^{37}$ Dept. of Physics, Engineering Physics, and Astronomy, Queen's University, Kingston, ON K7L 3N6, Canada

${ }^{38}$ Dept. of Physics and Astronomy, University of Kansas, Lawrence, KS 66045, USA

${ }^{39}$ Dept. of Physics and Astronomy, University of Nebraska-Lincoln, Lincoln, Nebraska 68588, USA

${ }^{40}$ Dept. of Physics, King's College London, London WC2R 2LS, United Kingdom

${ }^{41}$ School of Physics and Astronomy, Queen Mary University of London, London E1 4NS, United Kingdom

42 Department of Physics and Astronomy, UCLA, Los Angeles, CA 90095, USA

${ }^{43}$ Department of Physics, Mercer University, Macon, GA 31207-0001, USA

${ }^{44}$ Dept. of Astronomy, University of Wisconsin-Madison, Madison, WI 53706, USA

${ }^{45}$ Dept. of Physics and Wisconsin IceCube Particle Astrophysics Center, University of Wisconsin-Madison, Madison, WI 53706, USA

${ }^{46}$ Institute of Physics, University of Mainz, Staudinger Weg 7, D-55099 Mainz, Germany

${ }^{47}$ School of Physics and Astronomy, The University of Manchester, Oxford Road, Manchester, M13 9PL, United Kingdom

${ }^{48}$ Department of Physics, Marquette University, Milwaukee, WI, 53201, USA

${ }^{49}$ Institut für Kernphysik, Westfälische Wilhelms-Universität Münster, D-48149 Münster, Germany

${ }^{50}$ Bartol Research Institute and Dept. of Physics and Astronomy, University of Delaware, Newark, DE 19716, USA

${ }^{51}$ Dept. of Physics, Yale University, New Haven, CT 06520, USA

${ }^{52}$ Columbia Astrophysics and Nevis Laboratories, Columbia University, New York, NY 10027, USA

${ }^{53}$ Dept. of Physics, University of Notre Dame du Lac, 225 Nieuwland Science Hall, Notre Dame, IN 46556-5670, USA

${ }^{54}$ Dept. of Physics, University of Oxford, Parks Road, Oxford OX1 3PU, UK

${ }^{55}$ Dept. of Physics, Drexel University, 3141 Chestnut Street, Philadelphia, PA 19104, USA

${ }^{56}$ Physics Department, South Dakota School of Mines and Technology, Rapid City, SD 57701, USA

${ }^{57}$ Dept. of Physics, University of Wisconsin, River Falls, WI 54022, USA

${ }^{58}$ Dept. of Physics and Astronomy, University of Rochester, Rochester, NY 14627, USA

${ }^{59}$ Department of Physics and Astronomy, University of Utah, Salt Lake City, UT 84112, USA

${ }^{60}$ Oskar Klein Centre and Dept. of Physics, Stockholm University, SE-10691 Stockholm, Sweden

${ }^{61}$ Dept. of Physics and Astronomy, Stony Brook University, Stony Brook, NY 11794-3800, USA

62 Dept. of Physics, Sungkyunkwan University, Suwon 16419, Korea

${ }^{63}$ Institute of Basic Science, Sungkyunkwan University, Suwon 16419, Korea

${ }^{64}$ Earthquake Research Institute, University of Tokyo, Bunkyo, Tokyo 113-0032, Japan

65 Dept. of Physics and Astronomy, University of Alabama, Tuscaloosa, AL 35487, USA

${ }^{66}$ Dept. of Astronomy and Astrophysics, Pennsylvania State University, University Park, PA 16802, USA

${ }^{67}$ Dept. of Physics, Pennsylvania State University, University Park, PA 16802, USA

${ }^{68}$ Institute of Gravitation and the Cosmos, Center for Multi-Messenger Astrophysics, Pennsylvania State University, University Park, PA 16802, USA 
${ }^{69}$ Dept. of Physics and Astronomy, Uppsala University, Box 516, S-75120 Uppsala, Sweden

${ }^{70}$ Dept. of Physics, University of Wuppertal, D-42119 Wuppertal, Germany

${ }^{71}$ DESY, D-15738 Zeuthen, Germany

72 Università di Padova, I-35131 Padova, Italy

${ }^{73}$ National Research Nuclear University, Moscow Engineering Physics Institute (MEPhI), Moscow 115409, Russia

\section{Acknowledgements}

USA - U.S. National Science Foundation-Office of Polar Programs, U.S. National Science Foundation-Physics Division, U.S. National Science Foundation-EPSCoR, Wisconsin Alumni Research Foundation, Center for High Throughput Computing (CHTC) at the University of Wisconsin-Madison, Open Science Grid (OSG), Extreme Science and Engineering Discovery Environment (XSEDE), Frontera computing project at the Texas Advanced Computing Center, U.S. Department of Energy-National Energy Research Scientific Computing Center, Particle astrophysics research computing center at the University of Maryland, Institute for Cyber-Enabled Research at Michigan State University, and Astroparticle physics computational facility at Marquette University; Belgium - Funds for Scientific Research (FRS-FNRS and FWO), FWO Odysseus and Big Science programmes, and Belgian Federal Science Policy Office (Belspo); Germany - Bundesministerium für Bildung und Forschung (BMBF), Deutsche Forschungsgemeinschaft (DFG), Helmholtz Alliance for Astroparticle Physics (HAP), Initiative and Networking Fund of the Helmholtz Association, Deutsches Elektronen Synchrotron (DESY), and High Performance Computing cluster of the RWTH Aachen; Sweden - Swedish Research Council, Swedish Polar Research Secretariat, Swedish National Infrastructure for Computing (SNIC), and Knut and Alice Wallenberg Foundation; Australia - Australian Research Council; Canada - Natural Sciences and Engineering Research Council of Canada, Calcul Québec, Compute Ontario, Canada Foundation for Innovation, WestGrid, and Compute Canada; Denmark - Villum Fonden and Carlsberg Foundation; New Zealand Marsden Fund; Japan - Japan Society for Promotion of Science (JSPS) and Institute for Global Prominent Research (IGPR) of Chiba University; Korea - National Research Foundation of Korea (NRF); Switzerland - Swiss National Science Foundation (SNSF); United Kingdom - Department of Physics, University of Oxford. 